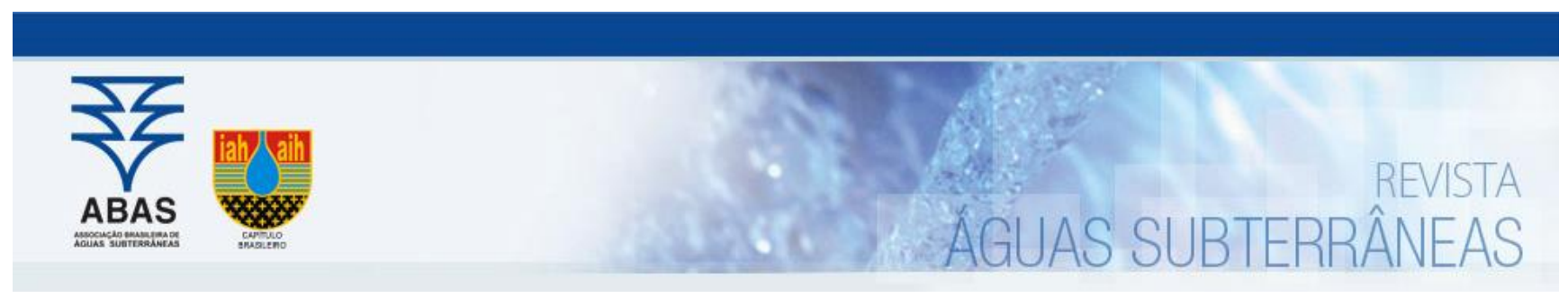

Artigos

\title{
Medidores de baixo custo para a quantificação de fluxo e coleta de amostras de água na interface leito/sedimentos em rios de montanha
}

\section{Low-cost seepage meters for flow measurement and water sampling in stream bed/sediments interface of mountain rivers}

\author{
Victor Alves Carias ${ }^{1}$; Mateus Ricardo Nogueira Vilanova ${ }^{1 凶}$ \\ 1 Universidade Estadual Paulista "Júlio de Mesquita Filho" (UNESP), São José dos Campos, SP. \\ victor.a.carias@gmail.com,mateus.vilanova@unesp.br
}

Palavras-chave:

Hidrologia de montanha. Interação rio-aquífero. Escoamento de base.

\begin{abstract}
Resumo
O estudo do fluxo hiporreico ganhou atenção nas últimas décadas, devido ao reconhecimento dos diversos processos físicos, geoquímicos e biológicos que ocorrem em sua interface. Estes influenciam, direta e indiretamente, os ecossistemas e a disponibilidade de recursos hídricos. A importância e a complexidade da zona hiporreica é aumentada em rios de montanha, localizados em regiões de alta relevância conservacionista e grande variabilidade hidrometeorológica. Apesar disso, a utilização de medidores de fluxo (seepage meters) tradicionais pode ser inviável nesse tipo de rio, devido às suas características físicas (composição granulométrico e geometria do leito) e pela dificuldade de acesso a certas áreas montanhosas. Diante do exposto, este trabalho reporta o desenvolvimento e a avaliação (laboratorial e in loco) de dois tipos de medidores de fluxo de baixo custo, construídos com tubos de PVC e baldes industriais. Uma bancada experimental com controle/medição de vazão, simulando um leito fluvial, foi desenvolvida para os ensaios, que tiveram seus resultados avaliados a partir do cálculo da da incerteza-padrão Tipo "A". Os testes laboratoriais realizados permitiram concluir que o medidor construído com balde industrial (M2) pode ser utilizado em medições reais, enquanto o medidor feito com tubo de PVC (M1) não apresentou bom desempenho. Durante os testes, o M2 apresentou um desvio de aproximadamente $12 \%$ em relação à vazão de referência da bancada, com uma incerteza padrão de 0,40 L. $\mathrm{m}^{-2}$.
\end{abstract}

\section{Keywords:}

Mountain hydrology.

River-aquifer interaction.

Baseflow.

Revisado por pares.

Recebido em: 23/09/2019.

Aprovado em: 20/04/2020.

\section{Abstract}

The study of hyporheic flow has gained attention in recent decades, due to the recognition of the various physical, geochemical and biological processes that occur at its interface. These processes directly and indirectly influence ecosystems and the water resources availability. The importance and complexity of the hyporheic zone is increased in mountain rivers, located in regions of high conservation relevance and great hydrometeorological variability. Nevertheless, the use of traditional seepage meters may be unfeasible in this type of river, due to their physical characteristics (bed sediments size and bed geometry) and the difficulty of access to certain mountainous areas. Given the above, this paper reports the development and evaluation (in laboratory and in loco) of two types of low-cost seepage meters, built with PVC pipes and industrial buckets. An experimental bench with discharge control/measurement, simulating a river bed, was developed for the tests, whose results were evaluated based on the calculation of the Type "A" standard uncertainty. The tests showed that the meter built with industrial bucket (M2) can be used in real measurements, while the meter made with PVC pipe (M1) did not perform well. During the tests, the M2 presented a deviation of approximately $12 \%$ in relation to the bench reference discharge, with a standard uncertainty of $0.40 \mathrm{~L} . \mathrm{m}-2$.

DOI: http:/dx.doi.org/10.14295/ras.v34i2.29619

\section{INTRODUÇÃO}

A zona hiporreica $(\mathrm{ZH})$ é constituída pelo volume de sedimentos no qual a água superficial de um rio se mistura com a água subterrânea (PERALTA-MARAVER; REISS; ROBERTSON, 2018). $A$ diversidade de definições de $\mathrm{ZH}$ se deve à pluralidade de disciplinas necessárias para o seu completo entendimento, utilizando abordagens geoquímicas, hidrológicas, biológicas e integradas, conforme Peralta-Maraver et al. (2018). Citando outros autores, Song et al. (2017) caracterizam a ZH como uma zona saturada ou cinemática conectando as águas subterrâneas às superficiais, que afeta e regula a dinâmica física e os processos biogeoquímicos de troca.

Trabalhos recentes sobre a ZH abordam, dentre outros, o fluxo de metais pesados em relação ao escoamento vertical e granulometria dos sedimentos (LIU et al., 2017), a quantificação de erros na determinação de fluxos verticais utilizando modelos analíticos unidimensionais (LU et al., 2017), os efeitos de inundações experimentais sobre comunidades de invertebra- 
dos em canais artificiais alimentados com águas residuais tratadas (BARANOV et al., 2017), a investigação da melhora da remoção de nutrientes em leitos fluviais arenosos e com cascalho através do acoplamento entre variação do fluxo vertical e transformações mediadas por micróbios (LI et al., 2017), a interação entre a dinâmica do escoamento superficial com a concentração de oxigênio dissolvido na ZH (KAUFMAN et al., 2017), a relação entre textura dos sedimentos, bioquímica e atividade biológica (HOU et al., 2017), e a influência das diferenças hidrológicas e físico-químicas entre as zonas bentônica e hiporreica na decomposição microbiana das folhas e na estrutura e função da comunidade microbiana associada (RISSE-BUHL et al., 2017).

Segundo Rosenberry (2008), quantificar a troca de água subterrânea e superficial na ZH ganhou importância nas últimas décadas, tanto para o gerenciamento dos recursos hídricos quanto de ecossistemas aquáticos, devido ao maior entendimento dos complexos processos físicos, químicos e biológicos que ocorrem nesta interface. Dessa forma, torna-se fundamental a utilização de dispositivos que permitam quantificar, in situ, a troca de água entre os cursos d’água e os aquíferos, produzindo dados observacionais para a análise e modelagem destes sistemas. Os principais métodos de campo para a medição do fluxo entre águas superficiais e subterrâneas, de acordo com Rosenberry et al. (2008), são: imageamento aéreo infravermelho, perfilamento térmico, sondas de temperatura e condutância específica, corantes e traçadores, potenciomanometros hidráulicos (mini-piezômetros), medidores de fluxo (seepage meters), redes de poços de observação e medições de vazão.

Os medidores de fluxo na interface leito/sedimentos (seepage meters) foram desenvolvidos, inicialmente, para realizar a medição de perdas de água em canais de irrigação (ROSENBERRY; LABAUGH; HUNT, 2008), sendo compostos, geralmente, por um tambor cortado, que isola parte do leito fluvial, e coleta o volume escoado na interface através de um saco plástico (ROSENBERRY; LABAUGH; HUNT, 2008). Um dos modelos mais conhecidos de medidor de fluxo foi proposto por Lee (1977), sendo composto pela seção final de um tambor de aço cilíndrico (volume total do tambor de 208 litros), revestido de epóxi, com diâmetro de $57 \mathrm{~cm}$ (área de coleta de aproximadamente $0,25 \mathrm{~m}^{2}$ ). Algumas variações e alternativas ao modelo clássico de Lee (1977) são apresentadas, por exemplo, por Rosenberry (2008).

Devido ao baixo custo e fácil operação, medidores pontuais de troca de água na interface água-sedimento constituem uma importante ferramenta para o estudo da zona hiporreica, podendo ser utilizados em rios, lagos, estuários e oceanos (LEE, 1977; ROSENBERRY, 2008). No Brasil, trabalhos como os de Arantes et al. (2006) e Arcari et al. (2019) utilizaram medidores de fluxo para quantificar as trocas entre águas superficiais e subterrâneas no Ribeirão da Onça (Brotas-SP) e na Lagoa da Conceição (Florianópolis-SP), respectivamente.

Apesar de úteis e aplicáveis em diversas situações, os see- page meters convencionais podem ser operacional e logisticamente inadequados para o monitoramento de pequenos corpos d'água, sobretudo em regiões montanhosas. Esse tipo de região apresenta grande relevância hidrológica e ecológica, devido à sua intensa dinâmica hidrometeorológica, e à presença de ecossistemas típicos e vulneráveis. Os rios de montanha são, geralmente, estreitos e com grande declividade, com leito composto de material de elevada granulometria, incluindo seixos e matacões.

Nesse tipo de condição, a instalação de medidores de fluxo convencionais (fabricados com tambores metálicos de grandes dimensões), pode ser inviável, devido à dificuldade de cravamento no leito, e mesmo à dificuldade de transporte até o local da instalação. Apesar de medidores de grande diâmetro serem mais precisos em condições de baixo fluxo, e permitirem uma melhor caracterização em pequena escala, seu peso e a dificuldade de garantir boa vedação em leitos desiguais e rochosos (ROSENBERRY; LABAUGH; HUNT, 2008) comprometem seu uso em rios de montanha. Além disso, apesar de o custo unitário destes medidores ser relativamente baixo, a construção de várias unidades pode ser onerosa, o que reduz a viabilidade de sua instalação em diversos pontos de um rio, para a análise da variabilidade espacial do fluxo na ZH.

A relevância hidrológica e ecológica de rios de montanha, e as dificuldades de se utilizar seepage meters convencionais nesse tipo de rio, motivaram o desenvolvimento da presente pesquisa, que desenvolveu e testou duas alternativas portáteis e de baixo custo para a medição das trocas de água no leito desse tipo de rio.

\subsection{Evolução histórica dos medidores de fluxo}

O desenvolvimento de medidores de fluxo de troca de águas subterrâneas para a superfície teve início em 1944, quando um dispositivo foi desenvolvido para a medição da perda de água em canais de irrigação (ISRAELSON; REEVE, 1944). Esse dispositivo era formado por um cilindro inferior coberto por um cone com uma válvula, um cilindro superior, um saco de material fino com água, sendo este impermeável e flexível, além de uma mola elástica sustentada por um grampo.

Ainda em termos históricos, Carr e Winter (1980) apresentaram uma importante compilação anotada de trabalhos pioneiros no desenvolvimento de seepage meters.

Os dispositivos desenvolvidos inicialmente tinham alto custo (ROSENBERRY; LABAUGH; HUNT, 2008), o que levou Lee (1977) a propor a construção de um medidor de baixo custo para a realização de medições de fluxo em lagos e estuários, sem a necessidade de medição da permeabilidade dos sedimentos. Para isso, Lee (1977) utilizou um galão de aço de 208 litros cortado ao meio, no qual foi acoplado um saco plástico coletor parcialmente preenchido de água, com volume conhecido. 0 saco coletor é fixado ao tambor e o volume de água acumulado é, então, medido. O arranjo proposto por Lee (1977) é apresentado na Figura 1. 
Figura 1 - Medidor de fluxo na interface rio-sedimento proposto por Lee (1977)

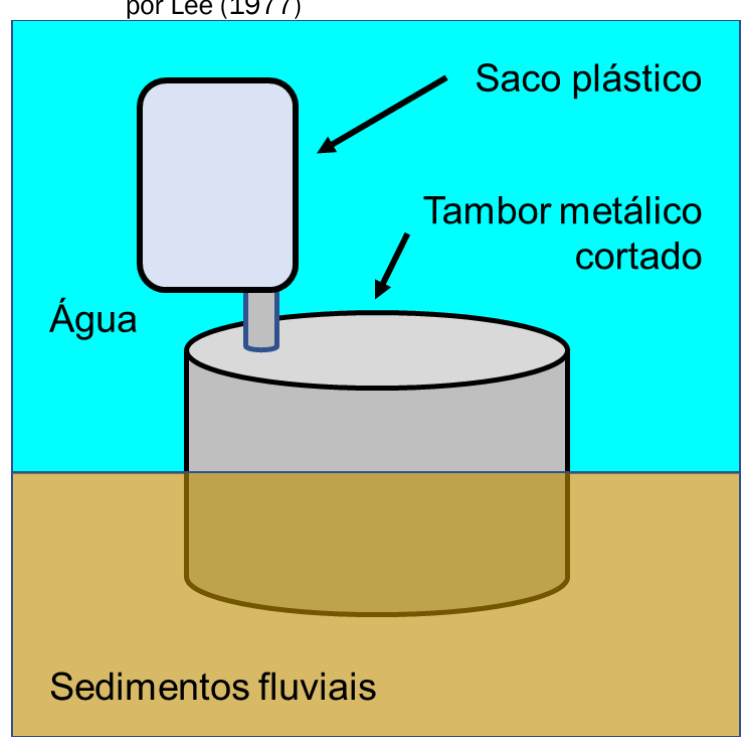

Fonte: adaptado de Lee (1977)

Utilizando-se o medidor de Lee (1977), divide-se a variação do volume de água armazenada no saco coletor pelo tempo de coleta, obtendo-se uma taxa de fluxo volumétrico (vazão, em unidade de $\mathrm{m}^{3} \cdot \mathrm{s}^{-1}$, por exemplo), que escoa através da interface rio-aquífero na área delimitada pelo medidor. A troca de água na interface é comumente expressa em termos unitários, dividindo-se a taxa de fluxo volumétrico $\left(\mathrm{m}^{3} . \mathrm{s}^{-1}\right)$ pela área da seção transversal do medidor $\left(\mathrm{m}^{2}\right)$, resultando em valores na dimensão de velocidade de fluxo (m.s-1) (LEE, 1977; ROSENBERRY; LABAUGH; HUNT, 2008).

A evolução dos seepage meters se deu pela necessidade da sua utilização diferentes ambientes e condições (ROSENBERRY; MENHEER, 2006), incluindo nas proximidades das margens fluviais (LEE; CHERRY, 1979), em águas profundas (BOYLE, 1994) e em locais em que o medidor era exposto a forças em que precisaria resistir devido a correntes ou ondas (CHERKAUER; MCBRIDE, 1988). O modelo de medidor proposto por Lee (1977) também foi utilizado para a realização de análises sobre a influência sazonal, para a quantificação da descarga de águas subterrâneas em baixas e altas marés (DEBNATH; MUKHERJEE, 2016).

Diversos tipos e tamanhos de tambores foram utilizados ao longo dos anos. Além disso, surgiram discussões sobre o tamanho ideal e tipos de sacos plásticos que são acoplados ao tambor (ROSENBERRY; MENHEER, 2006). Tubos de pequenos diâmetros (compostos de metal, vidro ou plástico), acionados através de tampas de borracha para a conexão do saco coletor ao cilindro de infiltração eram utilizados nos dispositivos pioneiros (ROSENBERRY; MENHEER, 2006). Contudo, uma resistência substancial ao fluxo passou a ser verificada, acarretando em uma ineficiência ao medir taxas mais altas de escoamento (FELLOWS; BREZONIK, 1980; ROSENBERRY; MORIN, 2004). Numa tentativa de reduzir essa ineficiência, fatores de correção para os medidores foram determinados em vários projetos destes dispositivos, através da comparação entre as taxas de escoamento que foram geradas em um tanque de infiltração e as taxas registradas pelos medidores.

Atualmente, diversos pesquisadores adotaram tubos com diâmetros maiores na conexão com os sacos coletores, no intuito de aumentar a eficiência do dispositivo de medição (HARVEY et al., 2000; ROSENBERRY, 2005; ROSENBERRY; MORIN, 2004). Ademais, foram desenvolvidos outros tipos de medidores automatizados, que não requerem o uso de um saco coletor (HARVEY et al., 2000; MENHEER, 2004; PAULSEN et al., 2001; ROSENBERRY; MORIN, 2004; SHOLKOVITZ; HERBOLD; CHARETTE, 2011; TANIGUCHI; IWAKAWA, 2001; TRYON et al., 2001; ZHU et al., 2015).

\section{MATERIAL E MÉTODOS}

\subsection{Construção dos medidores de fluxo}

Foram testados dois tipos de medidores: o primeiro (M1) foi construído com tubo de PVC, e o segundo (M2) com balde plástico industrial (encontrado comumente em lojas de embalagens), conforme Figura 2. 
Figura 2 - Esquema do arranjo básico dos medidores propostos

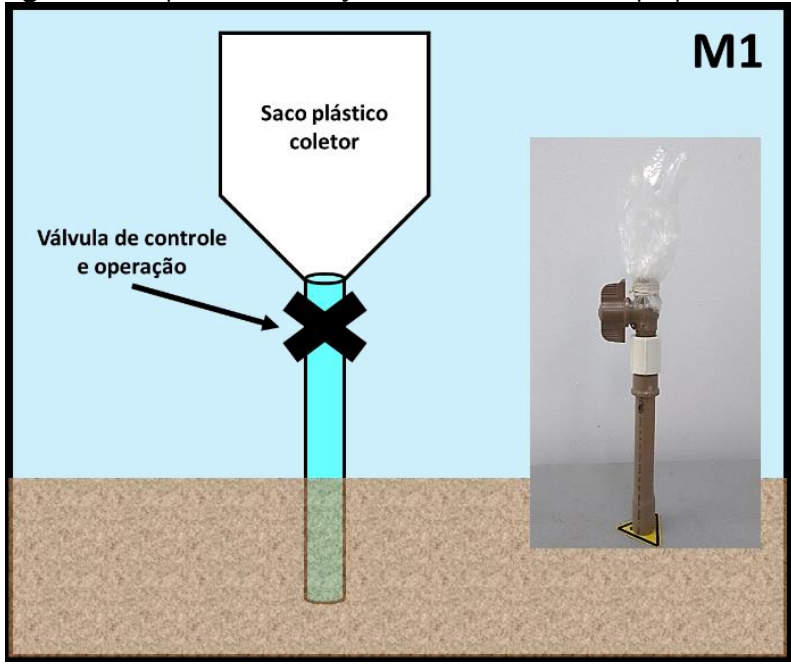

O M1 foi construído com tubo de PVC, com diâmetro nominal de $20 \mathrm{~mm}$, sendo seu comprimento de $20 \mathrm{~cm}$ a $30 \mathrm{~cm}$, permitindo um cravamento mínimo $15 \mathrm{~cm}$ na camada de sedimentos. O M2 foi construído com balde plástico industrial, com capacidade aproximada de $3 \mathrm{~L}$ (diâmetro de $180 \mathrm{~mm}$ ).

Os dois medidores foram acoplados a um saco plástico para armazenamento de alimentos, que foi devidamente vedado para evitar a infiltração de qualquer outra fonte de água que não seja originada do fluxo água-sedimento. Antes dos ensaios, os sacos foram submetidos a testes de estanqueidade, para verificar a ausência de furos. Entre o tubo e o saco coletor foi instalada uma válvula de controle, cujo objetivo é reter um volume inicial de água no início da operação do medidor, e

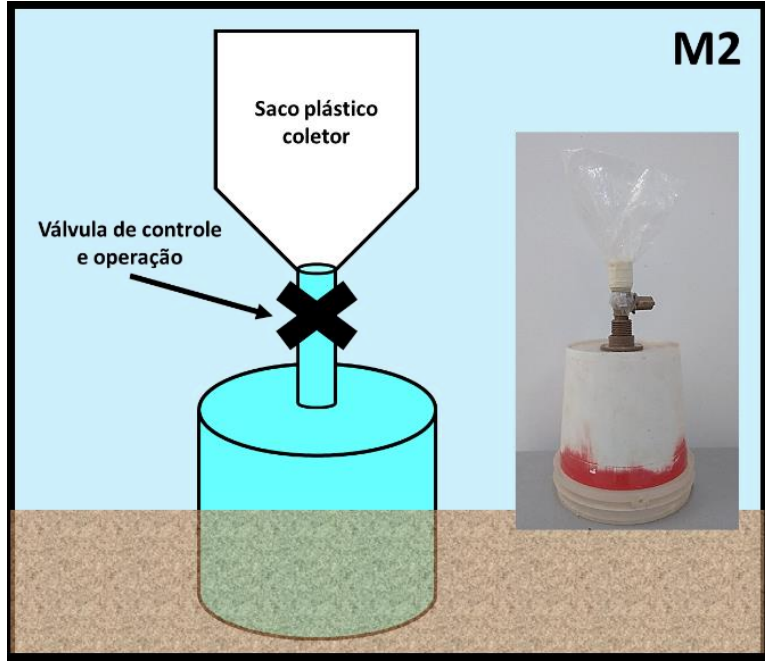

isolar o volume final coletado logo após o término do experimento, conforme descrito na seção 2.3 (ROSENBERRY; LABAUGH, 2008).

\subsection{Arranjo experimental laboratorial}

0 arranjo experimental laboratorial para a análise dos medidores propostos se baseou nos trabalhos de Solder (2014) e Solder et al. (2016). O arranjo proposto e adaptado é representado na Figura 3, que considera apenas o arranjo para o M1 (o arranjo para o M2 é idêntico, mudando apenas o medidor). 0 arranjo experimental utilizado é apresentado na Figura 4.

Figura 3 - Esquema do arranjo experimental laboratorial

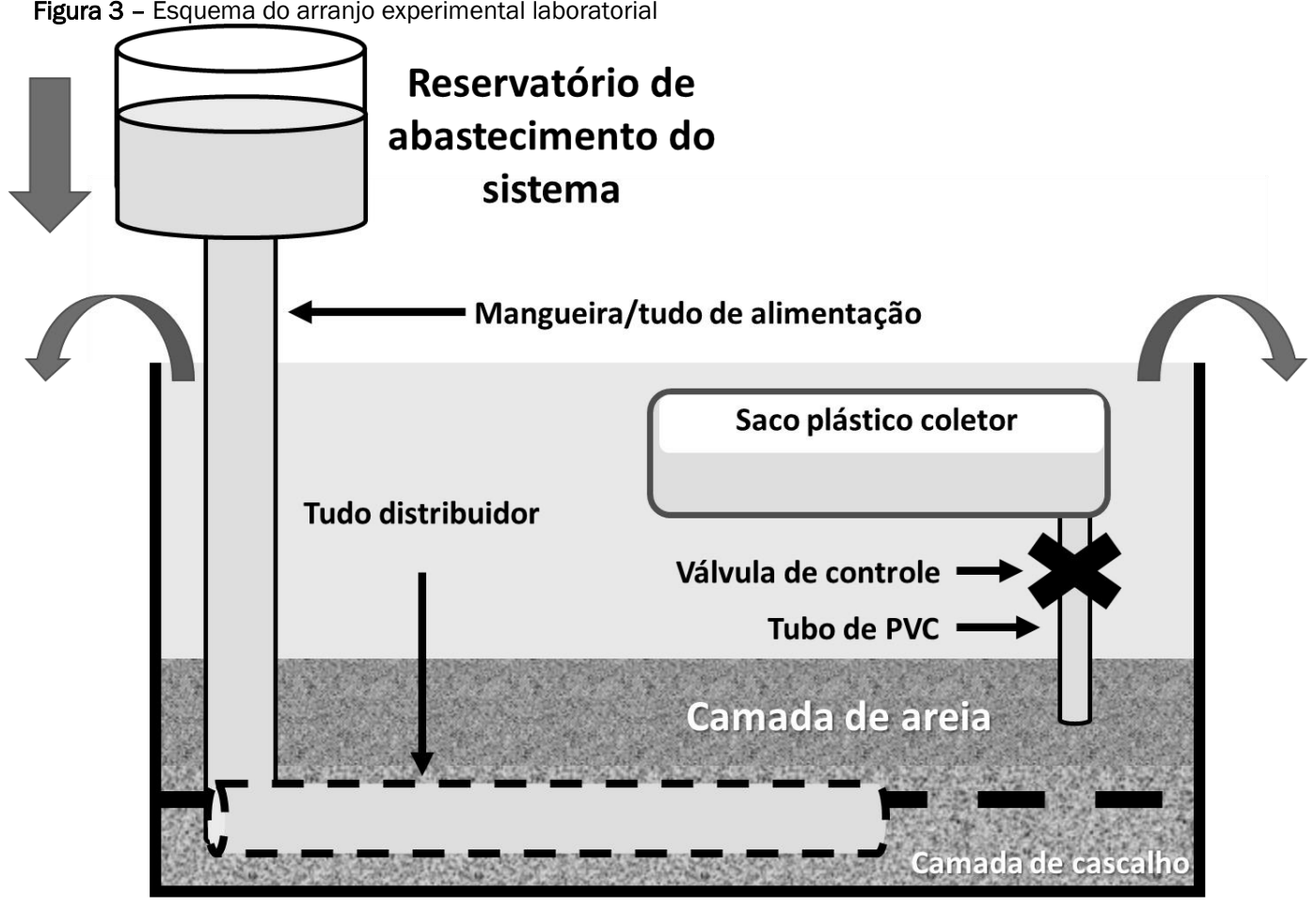


Figura 4 - Arranjo experimental laboratorial: a) Tanque de alimentação; b) tubo distribuidor; c) cobertura de cascalho; d) cobertura de areia; e) arranjo experimental completo
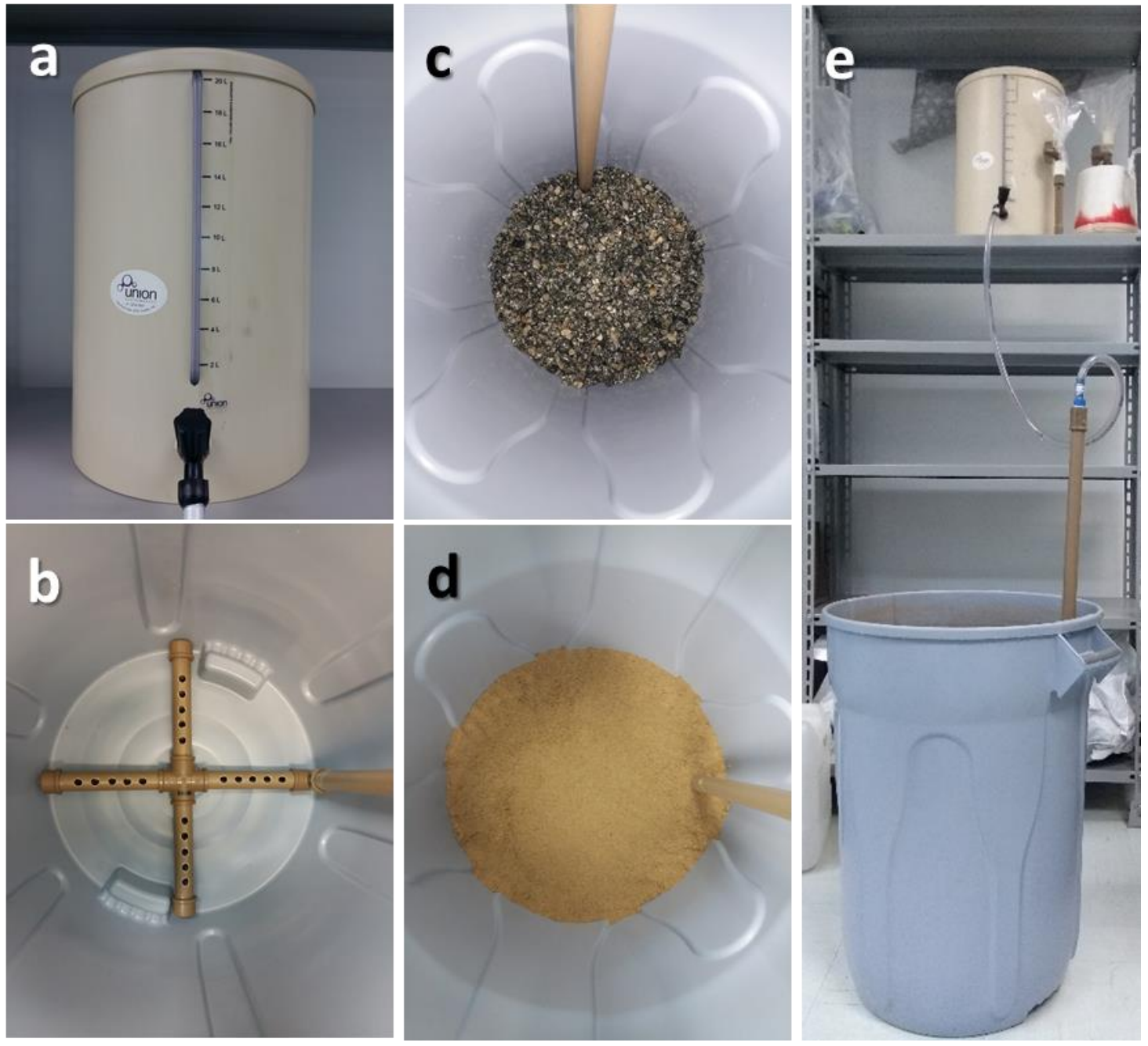

O tanque que simulou o corpo d’água foi constituído de um balde de 100 litros. Foi utilizado um tanque graduado (originalmente utilizado em um permeâmetro de carga constante) como reservatório de alimentação do sistema. 0 tubo alimentador foi posicionado no fundo do tanque, e envolto por uma manta têxtil, e então coberto por uma camada de cascalho que, teoricamente, garantiu um escoamento homogêneo em toda a área do tanque. A presença dessa manta protetora se fez necessária para impedir que parte do cascalho penetrasse no tubo de distribuição, e alterasse a homogeneidade do escoamento. 0 substrato simulando os sedimentos de rios de montanha foi composto de areia de filtro de piscina (granulometria de $0,5 \mathrm{~mm}$ a $1,0 \mathrm{~mm}$ ), que formou uma camada de sedimento de aproximadamente $25 \mathrm{~cm}$ de altura.

O volume unitário (volume por unidade de área da boca dos coletores) coletado nos sacos foi comparado com o volume unitário teórico. Esse volume teórico foi obtido através da razão entre o volume total escoado no tanque principal e área transversal total do substrato de areia.

\subsection{Operação dos medidores}

Para a operação dos medidores, deve-se considerar que o es coamento na interface rio-aquífero pode ocorrer bidirecionalmente, em função do gradiente hidráulico: do aquífero para o rio (situação na qual se caracteriza o rio como efluente, gaining), ou do rio para o aquífero (caracterizando o rio como influente, losing). Em função disso, diversos autores recomendam que os sacos coletores dos medidores de fluxo sejam parcialmente preenchidos com um volume inicial de água, antes do início da sua operação. Com este procedimento, na condição de rio influente, a diferença entre o volume inicial e final de água representa o escoamento absorvido pelo aquífero.

Uma vez preenchido o volume inicial de água ${ }^{1}$, retira-se o ar presente no saco coletor e fecha-se a válvula de controle, antes de se iniciar a instalação. 0 medidor é então fixado no ponto de interesse, através de cravamento nos sedimentos. Apesar de não haver um padrão, o cravamento do medidor deve ser profundo o suficiente para garantir a perfeita vedação da boca do equipamento, evitando a entrada de água proveniente do canal fluvial (escoamento superficial). A manobra de cravamento dos medidores nos sedimentos deve contemplar, também, uma manobra de eliminação do ar na cavidade do balde ou tubo de PVC. Essa manobra consiste em fechar a válvula do medidor já abastecido com o volume inicial, e mergulha-lo na água com a boca voltada para cima, de forma que

\footnotetext{
${ }^{1}$ No caso dos experimentos laboratoriais, foi utilizado o volume inicial de 0,2 litros.
} 
a sua cavidade seja completamente preenchida por água. Feito isso, a boca do medidor é direcionada para baixo (com o equipamento totalmente imerso), e então cravado no substrato do leito.

Após a instalação do medidor no sedimento, o reservatório de alimentação era acionado, dando início ao escoamento. A válvula de controle do medidor era, então, aberta no exato instante em que se lia o volume inicial do reservatório de alimentação e se disparava o cronômetro. 0 término de cada réplica do ensaio ocorria no instante no qual, simultaneamente, a válvula de controle do medidor era fechada, o volume final do reservatório de alimentação era medido, e o cronômetro parado.

Em ambos os medidores testados, foram analisados os volumes específicos escoados na interface dos sedimentos. Este volume corresponde a uma altura de escoamento, ou seja, volume por unidade de área ( $\mathrm{L} . \mathrm{m}^{-2}$ ou $\left.\mathrm{m}^{-3} \cdot \mathrm{m}^{-2}\right)$.

Os demais cuidados e procedimentos operacionais necessários para a garantia de qualidade dos dados seguiram as recomendações de Rosenberry et al. (ROSENBERRY; LABAUGH; HUNT, 2008).

\subsection{Cálculo da incerteza de medição}

Para a avaliação dos resultados das medições laboratoriais, foi utilizado o cálculo da incerteza das medições.

Ao se relatar o resultado da medição de uma grandeza física, faz-se necessário algum indicativo da qualidade do resultado, uma vez que, quando utilizado por terceiros, a sua confiabilidade pode ser avaliada. Além disso, é possível que os resultados medidos sejam comparados com demais resultados ou valores de referência fornecidos por uma especificação ou norma. Nesse sentido, o presente trabalho considerou o cálculo da incerteza de medição.

A incerteza é definida como um parâmetro associado ao resultado de uma medição, que caracteriza a dispersão dos valores que podem ser razoavelmente atribuídos ao mensurado (JOINT COMMITTEE FOR GUIDES IN METROLOGY, 2008). O erro no resultado da medição tem origem em imperfeições na realização da medição, podendo ser classificado em diversos tipos como, aleatórios e sistemáticos.

A avaliação da incerteza-padrão Tipo "A" pode ser aplicada à uma situação em que várias observações independentes, feitas para uma grandeza qualquer de entrada, em que ocorre uma dispersão perceptível ou propagação no valor obtido, havendo resolução suficiente nesse processo de medição (ABBAS; KUMAR, 2019). No presente estudo, imperfeições na montagem, operação dos medidores e realização dos ensaios podem interferir no resultado da medição. Dessa forma, foi conduzida uma avaliação de incerteza padrão Tipo A (JOINT COMMITTEE FOR GUIDES IN METROLOGY, 2008).

Na medição de uma grandeza q, que varia de forma aleatória durante $n$ observações independentes realizadas sob as mesmas condições, a melhor estimativa disponível do valor esperado $\left(\mu_{q}\right)$ é a média aritmética $(\bar{q})$ destas observações, con- forme Equação 1 (JOINT COMMITTEE FOR GUIDES IN METROLOGY, 2008):

$\bar{q}=\frac{1}{n} \sum_{k=1}^{n} q_{k}$

Ao realizar uma série de observações individuais, as variações aleatórias podem resultar em resultados distintos (flutuação estatística), cuja flutuação pode ser expressa pela variância experimental $\left(s^{2}\left(q_{k}\right)\right)$ das observações, através da variância $\sigma^{2}$ da distribuição de probabilidade de $q$ (JOINT COMMITTEE FOR GUIDES IN METROLOGY, 2008) (Equação 2):

$s^{2}\left(q_{k}\right)=\frac{1}{n-1} \sum_{j=1}^{n}\left(q_{j}-\bar{q}\right)^{2}$

O desvio-padrão experimental $\left(s\left(q_{k}\right)\right)$ caracteriza a variabilidade dos valores $q_{k}$ observados e sua dispersão em torno da sua média $\bar{q}$ (JOINT COMMITTEE FOR GUIDES IN METROLOGY, 2008). A incerteza-padrão Tipo A $\left(U\left(\bar{q}_{k}\right)\right)$ do valor médio $q$, pode ser calculada pela Equação 3 (ABBAS; KUMAR, 2019; JOINT COMMITTEE FOR GUIDES IN METROLOGY, 2008):

$U\left(\bar{q}_{k}\right)=\frac{s\left(q_{k}\right)}{\sqrt{n}}$

\subsection{Estudo de caso in loco}

Considerando que os medidores propostos foram desenvolvidos para a aplicação em rios de montanha, após os testes em laboratório, foi realizado um experimento em campo. O local escolhido, que é representativo de uma bacia hidrográfica de montanha, foi o Córrego da Galhada, localizado no Parque Estadual de Campos do Jordão (Figura 5).

O clima da região é o Cfb na classificação de Köppen (subtropical temperado, sem estação seca) (GOVERNO DO ESTADO DE SÃO PAULO; SECRETARIA DE ESTADO DO MEIO AMBIENTE; FUNDAÇÃO FLORESTAL, 2015). Dados da estação pluviométrica código ANA 2245010 (VILANOVA, 2015), localizada em Campos do Jordão, indicam uma altura de chuva total anual média de $1.805 \mathrm{~mm}$, com totais sazonais médios de $620 \mathrm{~mm}$ na primavera, $790 \mathrm{~mm}$ no verão, $220 \mathrm{~mm}$ no outono e 175 $\mathrm{mm}$ no inverno. Em termos de balanço hídrico, a região de Campos do Jordão apresenta, em média, excedentes da ordem de $1179 \mathrm{~mm}$ por ano (GOVERNO DO ESTADO DE SÃO PAULO; SECRETARIA DE ESTADO DO MEIO AMBIENTE; FUNDAÇÃO FLORESTAL, 2015). A Bacia Hidrográfica do Córrego da Galhada (BHCG) é coberta, majoritariamente, por vegetação nativa preservada, no domínio da Mata Atlântica, sendo composta por Floresta Ombrófila Mista (Floresta de Araucárias), Floresta Ombrófila Densa Alto-montana (Mata Nebular), além de Campos de Altitude (GOVERNO DO ESTADO DE SÃO PAULO; SECRETARIA DE ESTADO DO MEIO AMBIENTE; FUNDAÇÃO FLORESTAL, 2015). A BHCG localiza-se no domínio geológico dos Complexos Granito-Gnaisse Migmatítico e Granulitos, com forma de relevo montanhoso (BRASIL, 2010). Predominam na Bacia os cambissolos (OLIVEIRA et al., 1999), cuja textura varia, em geral, de média a mais fina (INSTITUTO AGRONÔMICO DO ESTADO DE SÃO PAULO, 2016). A altimetria da bacia varia de aproximadamente $1.520 \mathrm{~m}$, no seu exutório (confluência com o Rio Sapucaí-Guaçu) a mais de $2.000 \mathrm{~m}$. 
Este estudo ocorreu através de 3 medições consecutivas, com duração de 15 mim a 20 min, tendo o medidor sido cravado nos sedimentos (predominantemente areia) em 3 pontos próximos ao indicado pela seta da Figura $6 a$.

\section{RESULTADOS E DISCUSSÃO}

\subsection{Experimento laboratorial}

A Tabela 1 apresenta o resultado dos ensaios com o M1.

Figura 5 - Estudo de caso: a) Visão geral do local de estudo e localização do medidor; b e c) vista subaquática do medidor durante as medições
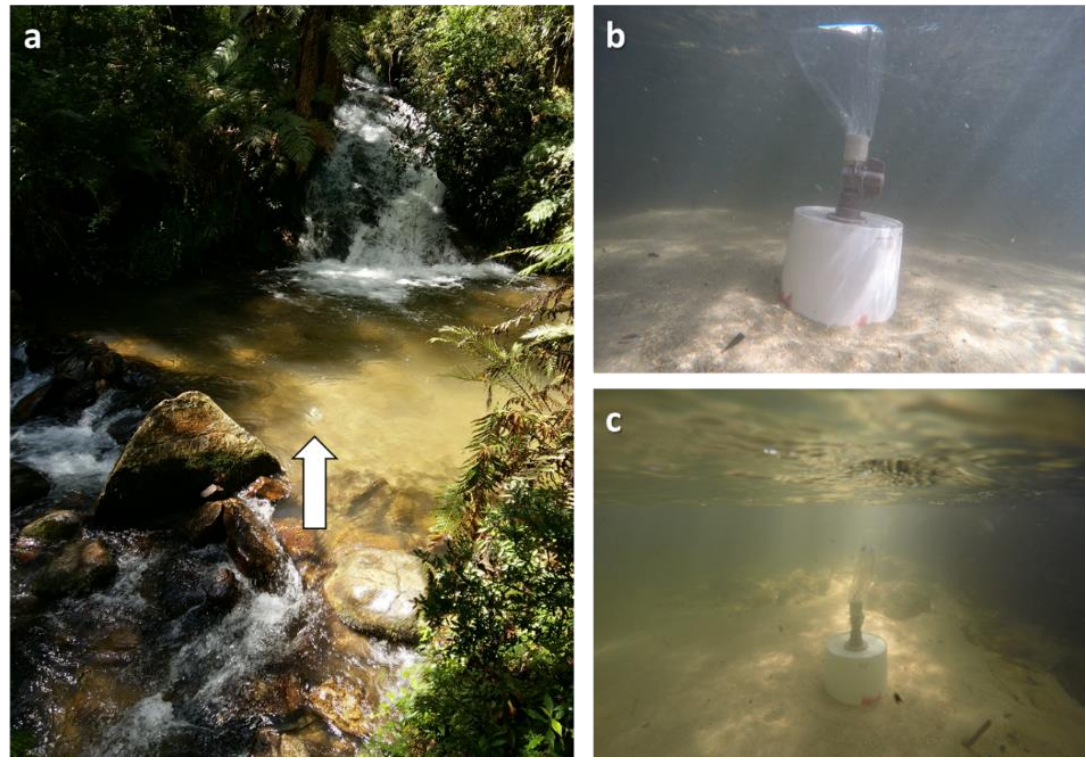

Tabela 1 - Resultados do experimento laboratorial com o medidor M1

\begin{tabular}{ccc}
\hline Ensaio $(\mathrm{n})$ & Medidor & $\begin{array}{c}\text { Volume unitário [L.m-2] } \\
\text { Reservatório principal }\end{array}$ \\
\hline 1 & 31,83 \\
2 & 63,66 \\
3 & 31,83 \\
4 & 50,93 \\
5 & 31,83 \\
Média $(\bar{q})$ & 42,02 \\
Desvio padrão amostral (s) & 14,66 \\
Variância experimental (s) & 214,80 \\
Incerteza padrão $(\mathbf{U})$ & 3,28 & - \\
\hline
\end{tabular}

Conforme verificado na Tabela 1, o medidor M1 apresentou um comportamento inesperado, resultando em uma grande diferença (da ordem de três vezes) do valor médio do volume unitário do medidor, correspondente a 42,02 L.m², em relação ao volume unitário apresentado pelo reservatório principal (14,08 L.m-2). Nesse sentido, o M1 superestimou os valores reais, apesar de apresentar uma incerteza padrão relativamente baixa, de 3,28 L.m-2. Uma hipótese para explicar este desempenho é a área da boca do M1, muito pequena quando comparada, por exemplo, com a do M2, tornando-a pouco representativa da variabilidade local do escoamento através do sedimento. Essa pequena área pode, também, causar alguma alteração no comportamento hidráulico do sedimento no local de inserção do medidor. Vários testes e réplicas foram realizados com o M1 (além daqueles descritos na Tabela 1), mas os resultados continuaram bastante discrepantes em relação ao valor esperado. Dessa forma, considerou-se o M1 inviável para aplicações reais.

A Tabela 2 apresenta os resultados dos ensaios com o M2. 
Tabela 2 - Resultados do experimento laboratorial com o medidor M2

\begin{tabular}{|c|c|c|}
\hline \multirow{2}{*}{ Ensaio (n) } & \multicolumn{2}{|c|}{ Volume unitário [L.m-2] } \\
\hline & Reservatório principal & Medidor \\
\hline 1 & & 11,99 \\
\hline 2 & & 12,97 \\
\hline 3 & & 14,15 \\
\hline 4 & & 13,36 \\
\hline 5 & & 10,61 \\
\hline 6 & & 10,22 \\
\hline 7 & & 12,18 \\
\hline 8 & & 10,22 \\
\hline 9 & & 13,75 \\
\hline 10 & & 15,13 \\
\hline 11 & $\perp 4,00$ & 13,75 \\
\hline 12 & & 11,79 \\
\hline 13 & & 9,82 \\
\hline 14 & & 9,82 \\
\hline 15 & & 10,61 \\
\hline 16 & & 12,97 \\
\hline 17 & & 13,56 \\
\hline 18 & & 14,15 \\
\hline 19 & & 15,72 \\
\hline 20 & & 11,79 \\
\hline Média & $q^{-}$ & 12,43 \\
\hline Desvio padrão amostral & $\mathrm{s}$ & 1,80 \\
\hline Variância experimental & $\mathrm{s}^{2}$ & 3,23 \\
\hline Incerteza padrão & $U$ & 0,40 \\
\hline
\end{tabular}

O M2 apresentou desempenho satisfatório: o valor médio do volume unitário do medidor, 12,43 L. $\mathrm{m}^{-2}$ se diferenciou em, aproximadamente, $12 \%$ do valor do volume unitário do reservatório, com uma incerteza padrão correspondente de 0,40 L.m-2.

Ainda que pequena, alguns fatores podem explicar a incerteza ocasionada pelas imperfeições das medições com o M2, dentre eles: (1) retirada de ar do saco plástico coletor que não ocorre da mesma forma para cada ensaio; (2) erros de paralaxe envolvendo a marcação do volume escoado no reservatório e aferição dos volumes iniciais e finais em cada ensaio; (3) o local de cravamento do balde, que não foi exatamente o mesmo na realização dos ensaios. Em relação a alteração da hidráulica do sedimento, a cada vez que o medidor M2 era retirado, uma pequena parcela de sedimento era arrastada, decantando posteriormente, antes das próximas repetições do ensaio. Essa condição pode ocasionar a formação de pequenas bolhas de ar no interior do sedimento, alterando a pressão desse perfil, e interferindo no escoamento espontâneo da água (ROSENBERRY; LABAUGH; HUNT, 2008). Rosenberry et al. (2008) listaram possíveis fontes de erro na utilização desse tipo de medidor: vedação incompleta entre a câma- ra do medidor de infiltração e sedimentos; cilindros instáveis; tempo insuficiente entre a instalação do medidor e a primeira medição; procedimentos inadequados de fixação do saco; resistência do saco e água em movimento; vazamentos; erro de medição; câmara de medidor de infiltração flexível; tempo de fixação do saco insuficiente ou excessivo; acumulação de gás aprisionado.

A pequena incerteza, associada ao pequeno desvio da média dos ensaios em relação ao volume real, calculado para o reservatório principal, permitem concluir que o M2 pode ser aplicado em medições reais, obtendo resultados bastante aceitáveis.

\subsection{Estudo de caso}

A Tabela 3 apresenta o resultado dos ensaios em campo realizados com o M2, no Parque Estadual de Campos do Jordão. Devido ao baixo desempenho do M1 nos ensaios laboratoriais (tendo o medidor sido considerado inadequado para medições de fluxo), este medidor não foi utilizado nas medições do estudo de caso.

Tabela 3 - Resultados do estudo de caso no Córrego da Galhada

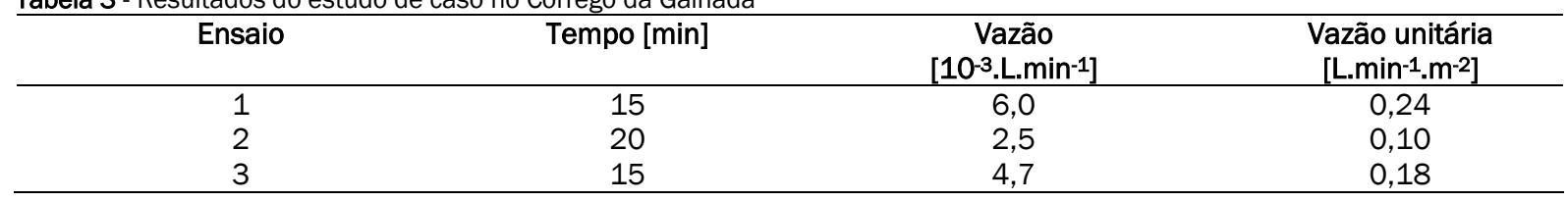

A vazão unitária média das 3 medições efetuadas no Córrego da Galhada, em 3 pontos próximos, foi da ordem de 0,17 L.min ${ }^{-1} \cdot \mathrm{m}^{-2}$, na condição de rio efluente (escoamento do aquífero para o rio). Este valor é uma primeira estimativa reportada na literatura sobre a troca de água nos leitos de rios da Serra da Mantiqueira (durante a revisão da literatura realizada para esta pesquisa, não foram encontrados trabalhos similares, realizados na Serra da Mantiqueira). Apesar deste ineditismo, o valor encontrado refere-se a ensaios pontuais (em termos espaciais e temporais), em determinados condições de armazenamento subterrâneo e de descarga na zona hiporreica. Para uma caracterização inicial representativa das trocas de água 
entre aquíferos e rios da Serra da Mantiqueira, são necessários ensaios semelhantes, porém, conduzidos durante longos períodos de tempo (para representar a variabilidade do escoamento ao longo do ano hidrológico) e em vários rios da Serra (para representar as diferentes características físicas/hidrogeológicas das suas bacias hidrográficas).

\section{CONCLUSÃO}

Este trabalho reportou o desenvolvimento e a avaliação de dois tipos de medidores de fluxo de baixo custo para o estudo do fluxo hiporreico em rios de montanha, construídos com tubos de PVC e baldes industriais. Com base nos resultados laboratoriais, o medidor M2 (construído com balde industrial) pode ser utilizado para medições reais do escoamento na interface rio-aquífero. Esse medidor apresentou um desvio de aproximadamente $12 \%$ em relação à vazão de referência da bancada, com uma incerteza padrão de 0,40 L.m-2. Essa incerteza correspondente a 3,2\% da vazão média dos ensaios. Em uma aplicação real em um rio de montanha (o Córrego da Galhada, localizado no Parque Estadual de Campos do Jordão), a vazão média obtida pelo M2 foi de 0,17 L. $\mathrm{min}^{-1} \cdot \mathrm{m}^{-2}$.

Os resultados desta pesquisa fornecem à comunidade hidrológica uma nova ferramenta para a coleta de dados de campo, demandados de forma cada vez mais intensa devido à necessidade de calibração e validação de modelos computacionais, e também para a gestão ambiental de bacias hidrográficas e entendimento dos efeitos hidrológicos das mudanças climáticas. Os custos dos componentes do M2 (que incluem balde plástico, saco plástico, anel adaptador de flange e válvula esfera) são de aproximadamente $\mathrm{R} \$ 35,00$. Além do baixo custo, este medidor é de fácil transporte e operação, sendo, portanto, ideal para a utilização em rios de montanha.

Considerando a expansão de plataformas abertas de hardware e software (como a plataforma Arduino), futuros trabalhos poderão propor e testar seepage meters de baixo custo automatizados, que permitirão avaliar o fluxo hiporreico de forma contínua, registrando, também, a sua variabilidade temporal.

Apesar de não indicado para a medição de fluxo, o medidor M1 pode constituir uma ferramenta bastante útil para a coleta de amostras de água na interface rio-aquífero.

\section{REFERÊNCIAS}

ABBAS, A.; KUMAR, A. Evaluation of uncertainty in flow and performance parameters in Francis turbine test rig. Flow Measurement and Instrumentation, v. 65, p. 297-308, jan./mar. 2019. https://doi.org/10.1016/j.flowmeasinst.2019.01.009

ARANTES, E. J.; CHAUDHRY, F. H.; MARCUSSI, F. F. N. Caracterização da interação entre rio e aqüífero. Águas Subterrâneas, v. 20, n. 2, p. 97-108, 1. fev. 2006. https://doi.org/10.14295/ras.v20i2.11728

ARCARI, T. D. L. et al. As interações entre águas subterrâneas e superficiais em uma laguna costeira: Lagoa da Conceição, Florianópolis/SC, Brasil. Águas Subterrâneas, v. 33, n. 1, p.
$34-44$,

26

jan.

2019.

https://doi.org/10.14295/ras.v33i1.29213

BARANOV, V. et al. Helophyte impacts on the response of hyporheic invertebrate communities to inundation events in intermittent streams. Ecohydrology, v. 10, n. 6, p. e1857, set. 2017. https://doi.org/10.1002/eco.1857

BOYLE, D. R. Design of a seepage meter for measuring groundwater fluxes in the nonlittoral zones of lakes-Evaluation in a boreal forest lake. Limnology and Oceanography, v. 39, n. 3, p. 670-681, maio 1994. https://doi.org/10.4319/lo.1994.39.3.0670

BRASIL, C. A. Geodiversidade do Estado de São Paulo. São Paulo: Serviço Geológico do Brasil, 2010.

CARR, M. R.; WINTER, T. C. An annotated bibliography of devices developed for direct measurement of seepage. Denver: [s.n.].

CHERKAUER, D. A.; MCBRIDE, J. M. A Remotely Operated Seepage Meter for Use in Large Lakes and Rivers. Ground Water, v. 26, n. 2, p. 165-171, mar. 1988. https://doi.org/10.1111/i.1745-6584.1988.tb00379.x

DEBNATH, P.; MUKHERJEE, A. Quantification of tidallyinfluenced seasonal groundwater discharge to the Bay of Bengal by seepage meter study. Journal of Hydrology, v. 537, p. 106-116, jun. 2016. https://doi.org/10.1016/j.jhydrol.2016.03.010

FELLOWS, C. R.; BREZONIK, P. L. SEEPAGE FLOW INTO FLORIDA LAKES. Journal of the American Water Resources Association, v. 16, n. 4, p. 635-641, ago. 1980. https://doi.org/10.1111/i.1752-1688.1980.tb02442.x

GOVERNO DO ESTADO DE SÃO PAULO; SECRETARIA DE ESTADO DO MEIO AMBIENTE; FUNDAÇÃO FLORESTAL. Parque Estadual de Campos do Jordão: plano de manejo. São Paulo. Disponivel em: http://www.ambiente.sp.gov.br/parquecampos-do-jordao/sobre-o-parque/.

HARVEY, J. W. et al. Interaction between ground water and surface water in the Northern Everglades and relation to water budgets and mercury cycling: Study methods and appendixesU.S. Geological Survey Open-File Report 00-168. Reston: [s.n.].

HOU, Z. et al. Geochemical and Microbial Community Attributes in Relation to Hyporheic Zone Geological Facies. Scientific Reports, v. 7, n. 1, p. 12006, 20 dez. 2017. https://doi.org/10.1038/s41598-017-12275-w

\section{INSTITUTO AGRONÔMICO DO ESTADO DE SÃO PAULO. Cambissolos. Disponível em: http://www.iac.sp.gov.br/solossp/pdf/Cambissolos.pdf.} Acesso em: 8 dez. 2016.

ISRAELSON, O. W.; REEVE, R. C. Bulletin No. 313 - Canal Lining Experiments in the Delta Area, UtahUAES Bulletins. Logan: [s.n.]. 
JOINT COMMITTEE FOR GUIDES IN METROLOGY. Evaluation of measurement data: guide to the expression of uncertainty in measurement. 1. ed. [s.I.] JCGM, 2008. v. 1

KAUFMAN, M. H. et al. Hyporheic hot moments: Dissolved oxygen dynamics in the hyporheic zone in response to surface flow perturbations. Water Resources Research, v. 53, n. 8, p. 6642-6662, ago.

2017.

https://doi.org/10.1002/2016WR020296

LEE, D. R. A device for measuring seepage flux in lakes and estuaries1. Limnology and Oceanography, v. 22, n. 1, p. 140147, jan. 1977. https://doi.org/10.4319/lo.1977.22.1.0140

LEE, D. R.; CHERRY, J. A. A Field Exercise on Groundwater Flow Using Seepage Meters and Mini-piezometers. Journal of Geological Education, v. 27, n. 1, p. 6-10, jan. 1979. https://doi.org/10.5408/0022-1368-27.1.6

LI, A. et al. Covariation in patterns of turbulence-driven hyporheic flow and denitrification enhances reach-scale nitrogen removal. Water Resources Research, v. 53, n. 8, p. 6927-6944, ago. 2017.

https://doi.org/10.1002/2016WR019949

LIU, Q. et al. Effects of Hyporheic Water Fluxes and Sediment Grain Size on the Concentration and Diffusive Flux of Heavy Metals in the Streambed. International Journal of Environmental Research and Public Health, v. 14, n. 9, p. 1020, 6 set. 2017. https://doi.org/10.3390/ijerph14091020

LU, C. P. et al. Differences in methods of quantifying the vertical hyporheic flow for streambank flow field. IOP Conference Series: Earth and Environmental Science, v. 82, n. 1, p. 012054, ago. 2017. https://doi.org/10.1088/1755$1315 / 82 / 1 / 012054$

MENHEER, M. A. Development of a benthic-flux chamber for measurement of ground-water seepage and water sampling for mercury analysis at the sediment-water interface. Reston: [s.n.].

OLIVEIRA, J. B. et al. Mapa Pedológico do Estado de São Paulo. Campinas: [s.n.].

PAULSEN, R. J. et al. Development and Evaluation of an Ultrasonic Ground Water Seepage Meter. Groundwater, v. 39, n. $6, \quad$ p. 904-911, nov. 2001. https://doi.org/10.1111/i.1745-6584.2001.tb02478.x

PERALTA-MARAVER, I.; REISS, J.; ROBERTSON, A. L. Interplay of hydrology, community ecology and pollutant attenuation in the hyporheic zone. Science of The Total Environment, v. 610611, p. 267-275, jan. 2018. https://doi.org/10.1016/j.scitotenv.2017.08.036

RISSE-BUHL, U. et al. Contrasting habitats but comparable microbial decomposition in the benthic and hyporheic zone. Science of The Total Environment, v. 605-606, p. 683-691, dez. 2017. https://doi.org/10.1016/i.scitotenv.2017.06.203
ROSENBERRY, D. O. Integrating seepage heterogeneity with the use of ganged seepage meters. Limnology and Oceanography: Methods, v. 3, n. 2, p. 131-142, fev. 2005. https://doi.org/10.4319/lom.2005.3.131

ROSENBERRY, D. O. A seepage meter designed for use in flowing water. Journal of Hydrology, v. 359, n. 1-2, p. $118-$ 130 , set. 2008.

https://doi.org/10.1016/i.jhydrol.2008.06.029

ROSENBERRY, D. O.; LABAUGH, J. W. Field techniques for estimating water fluxes between surface water and ground waterTechniques and Methods 4-D2. Reston: [s.n.].

ROSENBERRY, D. O.; LABAUGH, J. W.; HUNT, R. J. Use of monitoring wells, portable piezometers, and seepage meters to quantify flow between surface water and ground waterField Techniques for Estimating Water Fluxes Between Surface Water and Ground Water. Reston: [s.n.].

ROSENBERRY, D. O.; MENHEER, M. A. A system for calibrating seepage meters used to measure flow between ground water and surface water. Reston: [s.n.].

ROSENBERRY, D. O.; MORIN, R. H. Use of an Electromagnetic Seepage Meter to Investigate Temporal Variability in Lake Seepage. Ground Water, v. 42, n. 1, p. 68-77, jan. 2004. https://doi.org/10.1111/j.1745-6584.2004.tb02451.x

SHOLKOVITZ, E. R.; HERBOLD, C. W.; CHARETTE, M. A. An automated dye-dilution based seepage meter for the timeseries measurement of submarine groundwater discharge. Limnology and Oceanography: Methods, v. 1, p. 16-28, 2011. https://doi.org/10.4319/lom.2011.1.16

SOLDER, J. E. et al. A Tube Seepage Meter for In Situ Measurement of Seepage Rate and Groundwater Sampling. Groundwater, v. 54, n. 4, p. 588-595, jul. 2016. https://doi.org/10.1111/gwat.12388

SOLDER, J. E. E. Quantifying groundwater-surface water exchange: development and testing of shelby tubes and seepage blankets as discharge measurement and sample collection devices. [s.I.] University of Utah, 2014.

SONG, J. et al. Variability in the vertical hyporheic water exchange affected by hydraulic conductivity and river morphology at a natural confluent meander bend. Hydrological Processes, v. 31, n. 19, p. 3407-3420, 15 set. 2017. https://doi.org/10.1002/hyp.11265

TANIGUCHI, M.; IWAKAWA, H. Measurements of submarine groundwater discharge rates by a continuous heat-type automated seepage meter in Osaka Bay, Japan. Journal of Groundwater Hydrology, v. 43, n. 4, p. 271-277, 2001. https://doi.org/10.5917/jagh1987.43.271

TRYON, M. et al. A new benthic aqueous flux meter for very low to moderate discharge rates. Deep Sea Research Part I: Oceanographic Research Papers, v. 48, n. 9, p. 2121-2146, ago. 2001. https://doi.org/10.1016/S09670637(01)00002-4 
VILANOVA, M. R. N. Long-term rainfall trends in Serra da Mantiqueira Environmental Protection Area, southeast Brazil. Environmental Earth Sciences, v. 73, n. 8, p. 4779-4790, 12 abr. 2015. https://doi.org/10.1007/s12665-014-3763-y
ZHU, T. et al. Calibration and application of an automated seepage meter for monitoring water flow across the sedimentwater interface. Environmental Monitoring and Assessment, v. 187, n. 4, p. 171,10 abr. 2015. https://doi.org/10.1007/s10661-015-4388-7 